This item was submitted to Loughborough's Research Repository by the author.

Items in Figshare are protected by copyright, with all rights reserved, unless otherwise indicated.

\title{
An a posteriori error estimator for plane-strain geotechnical analyses
}

PLEASE CITE THE PUBLISHED VERSION

PUBLISHER

(C) Elsevier

VERSION

AM (Accepted Manuscript)

LICENCE

CC BY-NC-ND 4.0

\section{REPOSITORY RECORD}

El-Hamalawi, Ashraf, and M.D. Bolton. 2019. "An a Posteriori Error Estimator for Plane-strain Geotechnical Analyses”. figshare. https://hdl.handle.net/2134/5163. 
This item was submitted to Loughborough's Institutional Repository (https://dspace.lboro.ac.uk/) by the author and is made available under the following Creative Commons Licence conditions.

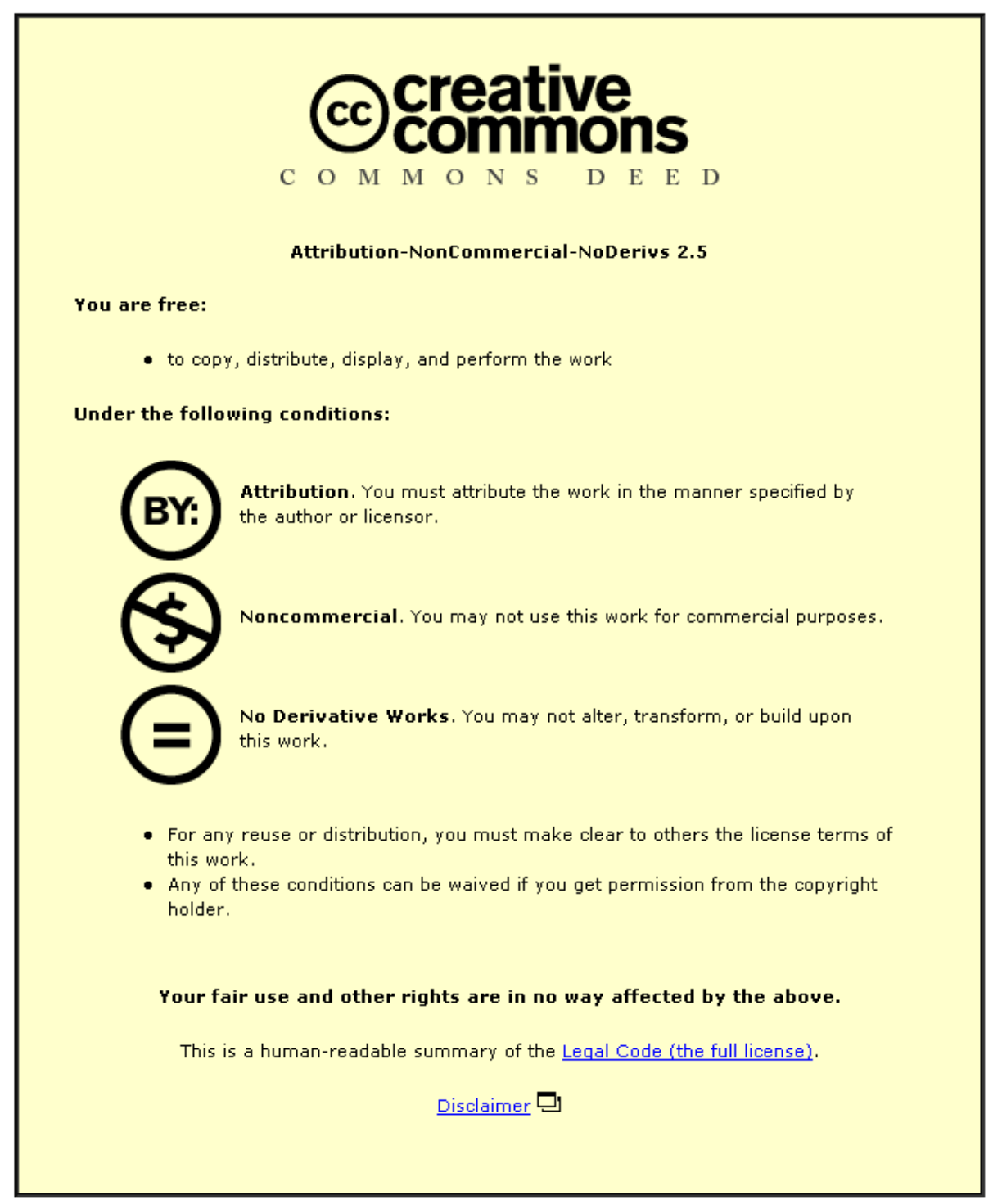

For the full text of this licence, please go to: http://creativecommons.org/licenses/by-nc-nd/2.5/ 


\section{An a-posteriori plane strain error estimator for geotechnical analyses}

\section{Introduction}

When a structure loads soil, causing the soil to yield, strains may be localised so that shear bands start to form. These bands influence the way in which the soil behaves and are usually indicative of incipient instability. It is highly desirable to be able to predict the progressive nature of the shear bands as they form due to strain localisation in a continuum. This modelling process is the subject of wide research. Special contact band elements have been used in regions where shear bands are expected to form by Wang et al. [1]. However, such an approach can only be followed for very simple problems where the position of localisations and direction of propagation can be assumed beforehand.

Regions of critical activity require a higher level of element discretization than elsewhere. One could make subjective assumptions regarding areas of high activity and use very small elements in these regions. However, making such judgements requires much experience and in most situations, such prior knowledge is lacking. This is especially true when analysing problems where soil behaviour is time-dependent. For soils undergoing consolidation, areas requiring refinement and de-refinement would be changing continuously with time, and this influences most geotechnical problems. Adaptive mesh refinement (AMR) therefore provides a suitable environment in which to model localisation, where no prior assumptions of the anticipated failure mechanisms or continuously changing regions in timedependent problems are required.

AMR in geomechanics is becoming popular, and has been in use since 1993 [2]. Mar analysed several undrained problems, using the $\mathrm{Z}^{2}$ error estimator [3]. Zienkiewicz et al. [4] managed to capture both ideal and softening plastic behaviour of the soil for two undrained problems. Hicks [5] analysed an undrained biaxial test, after using adaptive mesh refinement with an error estimator based on strains. However, the question of deriving an appropriate 
error criterion has not received sufficient attention. In particular, pore pressures are an essential facet of geotechnical analyses, and steep gradients magnify discretization errors. Many error estimation schemes exist, notably the SPR [6], SPREB [7] and more recently the REP [8] methods, which are in daily use with structural mechanics applications. However, these do not account for the dependency of pore pressures on time in consolidation-based problems, and the associated requirement for continuous changing element sizes.

An error criterion based on the SPREB method has been developed. This incorporates pore pressures and is therefore suitable for use in geotechnical engineering. Numerical examples have been described to demonstrate the effectiveness of this criterion in geotechnical engineering.

\section{Theory}

\subsection{The coupled consolidation governing equations}

In 1941, Biot [9] developed the theory of coupled consolidation, which has since evolved in many forms. The basic continuity equation is coupled with the equilibrium equations and converted to finite element matrix format. These are solved simultaneously, as shown below. This will be used in the next section to derive the equations upon which the consolidation patch recovery with equilibrium and boundary conditions (CSPREB) method is based.

Consider the two-dimensional equilibrium and continuity equations (1) and (2) respectively, over a domain $\Omega$ with boundary $\Gamma$. The boundary segment $\Gamma_{\sigma}$ is subject to $\mathrm{n} \cdot \sigma=\sigma_{b}$, and $\mathbf{u}=\mathbf{u}_{\mathbf{b}}$ acts on $\Gamma_{\mathbf{u}}$, where $\mathbf{n}$ is the outward unit vector, $\sigma$ is the stress vector, $\mathbf{u}$ the displacement vector and $\omega$ the body forces. The excess pore pressure $\sigma_{\mathrm{pp}}=\sigma_{\mathrm{ppb}}$, acts on the boundary segment $\Gamma_{\mathrm{pp}}$ at time $\mathrm{t}$, where $\mathrm{k}_{\mathrm{x}}$ and $\mathrm{k}_{\mathrm{y}}$ are the permeabilities in the $\mathrm{x}$ and $\mathrm{y}$ directions respectively, $\gamma_{\mathrm{w}}$ is the unit weight of water and $\varepsilon_{\mathrm{v}}$ is the volumetric strain. 


$$
\begin{gathered}
\mathrm{L}_{\text {eq }}^{\mathrm{T}} \sigma+\omega=0 \\
\frac{\mathrm{k}_{\mathrm{x}}}{\gamma_{\mathrm{w}}} \frac{\partial^{2} \sigma_{p p}}{\partial \mathrm{x}^{2}}+\frac{\mathrm{k}_{\mathrm{y}}}{\gamma_{\mathrm{w}}} \frac{\partial^{2} \sigma_{p p}}{\partial \mathrm{y}^{2}}+\frac{\partial \varepsilon_{\mathrm{v}}}{\partial \mathrm{t}}=0
\end{gathered}
$$

Sandhu and Wilson [10] were the first to introduce a composite displacement-pore pressure element, shown in figure 1, where the pore pressure variation was one order less than the displacement due to the stresses being derivatives of displacements. The pore pressures $\sigma_{\mathrm{pp}}$ are thus approximated using $\sigma_{\mathrm{pp}}=\mathrm{N}_{\mathrm{pp}} \bar{\sigma}_{\mathrm{pp}}$, where $\bar{\sigma}_{\mathrm{pp}}$ are the nodal pore pressures and $\mathrm{N}_{\mathrm{pp}}$ are the pore pressure shape functions.

Using the Galerkin weighted residual method, the product of equation (2) and an arbitrary function in the form of $\mathrm{N}_{\mathrm{pp}}$ is integrated by parts to result in equation (3), where $\mathrm{v}_{\mathrm{n}}$ is the seepage velocity normal to the boundary. Equation (4) shows the weak form of equation (2) after some mathematical simplification.

$$
\begin{gathered}
-\int_{\Omega}\left(\frac{\mathrm{k}_{\mathrm{x}}}{\gamma_{\mathrm{w}}} \frac{\partial \mathrm{N}_{\mathrm{pp}}}{\partial \mathrm{x}} \frac{\partial \sigma_{p p}}{\partial \mathrm{x}}+\frac{\mathrm{k}_{\mathrm{y}}}{\gamma_{\mathrm{w}}} \frac{\partial \mathrm{N}_{\mathrm{pp}}}{\partial \mathrm{y}} \frac{\partial \sigma_{p p}}{\partial \mathrm{y}}\right) d \Omega-\int_{\Gamma_{p p}} N_{p p}^{T} \mathrm{v}_{n} d \Gamma_{p p}+\int_{\Omega} N_{p p}^{T} \frac{\partial \varepsilon_{\mathrm{v}}}{\partial \mathrm{t}} d \Omega=0 \\
L_{p p}^{T} \frac{d \bar{u}}{d \mathrm{t}}-\Phi \bar{\sigma}_{p p}=\int_{\Gamma_{p p}} N_{p p}^{T} \mathrm{v}_{n} d \Gamma_{p p}
\end{gathered}
$$

where $L_{p p}=\int_{\Omega} B^{T} m N_{p p} d \Omega, \Phi=\int_{\Omega} \frac{E^{T} k E}{\gamma_{\mathrm{w}}} d \Omega, E=\left\{\begin{array}{c}\frac{\partial \mathrm{N}_{p p}}{\partial \mathrm{x}} \\ \frac{\partial \mathrm{N}_{p p}}{\partial \mathrm{y}}\end{array}\right\}$ and $k=\left[\begin{array}{cc}k_{x} & 0 \\ 0 & k_{y}\end{array}\right]$

To simplify the time differential, equation (4a) is integrated with respect to time from time $t$ to time $\mathrm{t}+\Delta \mathrm{t}$ using relation (5). Following Britto and Gunn [11] and based on Booker and Small [12], $\theta=1$ is assumed in order for the integration scheme to remain unconditionally stable.

$$
\int_{t}^{t+\Delta t} f . d t \cong\left((1-\theta) f_{1}+\theta f_{2}\right) \Delta t, \theta=1 \Rightarrow \int_{t}^{t+\Delta t} f . d t \cong f_{2} \Delta t=\left(f_{t+\Delta t}-f_{t}\right) \Delta t
$$




$$
L_{p p}^{T} \Delta \bar{u}-\Phi \Delta \mathrm{t} \Delta \bar{\sigma}_{p p}=\Phi \Delta \mathrm{t} \Delta \bar{\sigma}_{p p 1}+\int_{\Gamma_{p p}} N_{p p}^{T} \mathrm{v}_{n 2} \Delta \mathrm{t} d \Gamma_{p p}
$$

Upon coupling the result in equation (6) with the weak form of the equilibrium equations and writing in matrix format, the final finite element coupled consolidation equations are reduced to

$$
\left[\begin{array}{cc}
k & L_{p p} \\
L_{p p}^{T} & -\Phi \Delta \mathrm{t}
\end{array}\right]\left\{\begin{array}{c}
\Delta \bar{u} \\
\Delta \bar{\sigma}_{p p}
\end{array}\right\}=\left\{\begin{array}{c}
\Delta \mathrm{r}_{1} \\
\Delta \mathrm{r}_{2}
\end{array}\right\}
$$

where $\quad \Delta \mathrm{r}_{1}=\int_{\Omega} N_{u}^{T} w d \Omega+\int_{\Gamma_{\sigma}} N_{u}^{T} \sigma_{b} d \Gamma_{\sigma}$ and $\Delta \mathrm{r}_{2}=\Phi \Delta \mathrm{t} \Delta \bar{\sigma}_{p p 1}+\int_{\Gamma_{p p}} N_{p p}^{T} \mathrm{v}_{n 2} \Delta \mathrm{t} d \Gamma_{p p}$

The above equations are solved for the incremental displacements $\Delta \bar{u}$ and pore pressures $\Delta \bar{\sigma}_{p p}$, and marched forward in time to find a solution at time $t+\Delta t$ based on the solution at time t.

\subsection{The CSPREB method}

The consolidation superconvergent patch recovery method incorporating equilibrium and boundary conditions (CSPREB) is based on the SPREB method [7]. It involves fitting a least squares polynomial of one higher order than the shape functions for the displacements, and another for pore pressures at their optimal points in a patch of elements in order to obtain improved values. Smoothed variables such as strains, stresses and elasto-plasticity hardening parameters are then calculated.

Previous workers have shown that the SPR and SPREB methods are suitable for structural problems, where either pore pressures are non-existent, or are a function of the volumetric strain as in geotechnical undrained non-consolidation problems. However, the introduction of pore pressures as extra unknown primary variables during the finite element analysis necessitates some extra parameters. The consolidation problem analysed in the examples section demonstrates that although the pore pressures have a high deviation from 
the exact solution, the percentage displacement error tolerance is still satisfied, implying that no further refinement is necessary if the criteria was based solely on displacements.

Equation (2) is simplified by integrating with respect to time in order to eliminate the partial time derivative, resulting in :

$$
\frac{\Delta \mathrm{t}}{\gamma_{\mathrm{w}}}\left[\Delta\left(\mathrm{k}_{\mathrm{x}} \frac{\partial^{2} \sigma_{p p}}{\partial \mathrm{x}^{2}}+\mathrm{k}_{\mathrm{y}} \frac{\partial^{2} \sigma_{p p}}{\partial \mathrm{y}^{2}}\right)\right]+m^{\mathrm{T}} \varepsilon=0, m=\left\{\begin{array}{l}
1 \\
1 \\
1 \\
0
\end{array}\right\} \text { for 2D problems }
$$

where $\mathrm{m}^{\mathrm{T}} \varepsilon$ is the volumetric strain, and the $\Delta$ operator indicates an incremental approach.

The sum of the squares of the various residuals are defined by the functional $S$ :

$$
\begin{aligned}
S= & \frac{1}{2}\left[\sum_{I P} w_{1}^{2}\left(\sigma^{\prime *}-\sigma^{\prime}\right)^{2}+\sum_{\text {unod }} w_{2}^{2}\left(u^{*}-u\right)^{2}+\sum_{p p n o d} w_{3}^{2}\left(\sigma_{P P}^{*}-\sigma_{p p}\right)^{2}+\sum_{\text {bnod }} w_{4}^{2}\left(\mathrm{n} \cdot \sigma^{*}-\sigma_{b}\right)^{2}\right. \\
& \left.+\sum_{I P} w_{5}^{2}\left(L_{e q}^{T} \sigma^{*}+\omega\right)^{2}+\sum_{I P} w_{6}^{2}\left(\frac{\Delta t}{\gamma_{w}}\left[k_{x} \frac{\partial^{2} \sigma_{P P}^{*}}{\partial \mathrm{x}^{2}}+k_{y} \frac{\partial^{2} \sigma_{P P}^{*}}{\partial \mathrm{y}^{2}}\right]+m^{T} \varepsilon^{*}\right)^{2}\right]
\end{aligned}
$$

The functional comprises several component residuals, and weightings have been added to each residual in order to control their effect on the overall functional. The first three components constitute the effective stresses' residual at the reduced integration points for quadrilaterals (or optimum points for triangles), the displacements' residual at the displacement nodes, and the pore pressures' residual at the pore pressure nodes respectively. For the weightings of the displacement components $\mathrm{w}_{2}$, vertex nodes have a weighting of three or four times that of element internal nodes, as suggested by Wiberg and AbdulWahab [13]. Boundary fixed displacement and pore pressures nodes are also given a much higher weighting. The finite element solution satisfies essential boundary conditions, hence a high weighting is used in order to ensure that the improved displacements and pore pressures at the respective nodes are as close as possible, if not, equal to the actual boundary values. The fourth residual comprises the boundary traction conditions at the boundary nodes. It has 
however been found from numerical experimentation [14] that this condition may be too rigid a condition to satisfy.

The finite element method does not necessarily satisfy the equilibrium equations locally, but does globally. Included in the functional is an equilibrium equations' residual, which tries to satisfy equilibrium locally in the element patches in a least squares sense. One should note that for elasto-plastic problems, the equilibrium weighting should be set to zero. The last term in the functional is the continuity equation, which the CSPREB method tries as much as possible to satisfy in a local sense, rather than just globally.

Polynomials of one order higher than $\mathrm{N}_{\mathrm{u}}$ and $\mathrm{N}_{\mathrm{pp}}$ are assumed for the displacements $u^{*}=P_{a} \mathbf{a}$ and the pore pressures $\sigma_{p p}^{*}=P_{b} \mathbf{b}$ in the patch respectively, as shown in equation (11). $P_{\mathrm{a}}$ is therefore cubic and $P_{\mathrm{b}}$ is quadratic for quadratic displacement elements, where $P_{\mathrm{a}}$ and $P_{\mathrm{b}}$ are the co-ordinate terms' matrices and $\mathbf{a}$ and $\mathbf{b}$ are the unknown coefficient vectors. The smoothed effective stresses are substituted in the form $\sigma^{{ }^{*}}=\mathrm{DL}_{\varepsilon} u^{*}=\mathrm{DL}_{\varepsilon} P_{a} \mathbf{a}=\mathrm{Ma}$ where $\mathrm{M}=\mathrm{DL}_{\varepsilon} P_{a}, \mathrm{~L}_{\varepsilon}$ is the strain operator and $\sigma^{*}={\sigma^{\prime *}}^{*}+\sigma_{p p}^{*}$.

$$
u=\left\{\begin{array}{l}
u_{x} \\
u_{y}
\end{array}\right\}=P_{a} \mathbf{a}=\left[\begin{array}{cc}
P_{a} & 0 \\
0 & P_{a}
\end{array}\right]\left\{\begin{array}{l}
a_{x} \\
a_{y}
\end{array}\right\}, \sigma_{p p}=\left\{\begin{array}{c}
\sigma_{p p} \\
\sigma_{p p} \\
\sigma_{p p} \\
0
\end{array}\right\}=\left\{\begin{array}{c}
P_{b} \mathbf{b} \\
P_{b} \mathbf{b} \\
P_{b} \mathbf{b} \\
0
\end{array}\right\}
$$

The functional $S$ thus becomes:

$$
\begin{aligned}
S= & \frac{1}{2}\left[\sum_{I P} w_{1}^{2}\left(M \mathbf{a}-\sigma^{\prime}\right)^{2}+\sum_{\text {unod }} w_{2}^{2}\left(P_{a} \mathbf{a}-u\right)^{2}+\sum_{p p n o d} w_{3}^{2}\left(P_{b} \mathbf{b}-\sigma_{p p}\right)^{2}+\sum_{\text {bnod }} w_{4}^{2}\left(n\left(M \mathbf{a}+P_{b} \mathbf{b}\right)-\sigma_{b}\right)^{2}\right. \\
& \left.+\sum_{I P} w_{5}^{2}\left(L_{e q}^{T}\left(M \mathbf{a}+P_{b} \mathbf{b}\right)+\omega\right)^{2}+\sum_{I P} w_{6}^{2}\left(\frac{\Delta t}{\gamma_{w}}\left[k_{x} \frac{\partial^{2} P_{b} \mathbf{b}}{\partial \mathrm{x}^{2}}+k_{y} \frac{\partial^{2} P_{b} \mathbf{b}}{\partial \mathrm{y}^{2}}\right]+m^{T} L_{\varepsilon} P_{a} \mathbf{a}\right)^{2}\right]
\end{aligned}
$$

Minimising $S$ with respect to the two unknown coefficient vectors a and $\mathbf{b}$ and collecting terms: 


$$
\begin{aligned}
& \left(\begin{array}{l}
\sum_{I P} w_{1}^{2} M^{T} M+\sum_{\text {unod }} w_{2}^{2} P_{a}^{T} P_{a}+\sum_{\text {bnod }} w_{4}^{2}(n M)^{T}(n M) \\
+\sum_{I P} w_{5}^{2}\left(L_{e q}^{T} M\right)^{T}\left(L_{e q}^{T} M\right)+\sum_{I P} w_{6}^{2}\left(m^{T} L_{\varepsilon} P_{a}\right)^{T}\left(m^{T} L_{\varepsilon} P_{a}\right)
\end{array}\right)\left(\begin{array}{l}
\sum_{\text {bnod }} w_{4}^{2}(n M)^{T}\left(n P_{b}\right)+\sum_{I P} w_{5}^{2}\left(L_{e q}^{T} M\right)^{T}\left(L_{e q}^{T} P_{b}\right) \\
\mathbf{a}+\sum_{I P} w_{6}^{2}\left(m^{T} L_{\varepsilon} P_{a}\right)^{T}\left[\frac{\Delta t}{\gamma_{w}}\left[k_{x} \frac{\partial^{2} P_{b}}{\partial \mathrm{x}^{2}}+k_{y} \frac{\partial^{2} P_{b}}{\partial \mathrm{y}^{2}}\right]\right]
\end{array}\right) \mathbf{b} \\
& =\left[\sum_{I P} w_{1}^{2} M^{T} \sigma^{\prime}+\sum_{\text {unod }} w_{2}^{2} P_{a}^{T} u+\sum_{\text {bnod }} w_{4}^{2}(n M)^{T} \sigma_{b}+\sum_{I P} w_{5}^{2}\left(L_{e q}^{T} M\right)^{T} \omega\right] \\
& \left(\begin{array}{l}
\sum_{\text {bnod }} w_{4}^{2}\left(n P_{b}\right)^{T}(n M)+\sum_{I P} w_{5}^{2}\left(L_{e q}^{T} P_{b}\right)^{T}\left(L_{e q}^{T} M\right) \\
+\sum_{I P} w_{6}^{2}\left[\frac{\Delta t}{\gamma_{w}}\left[k_{x} \frac{\partial^{2} P_{b}}{\partial \mathrm{x}^{2}}+k_{y} \frac{\partial^{2} P_{b}}{\partial \mathrm{y}^{2}}\right]\right]^{T}\left(m^{T} L_{\varepsilon} P_{a}\right)
\end{array}\right)\left(\begin{array}{l}
\sum_{p \text { nnod }} w_{3}^{2} P_{b}^{T} P_{b}+\sum_{\text {bnod }} w_{4}^{2}\left(n P_{b}\right)^{T}\left(n P_{b}\right)+\sum_{I P} w_{5}^{2}\left(L_{e q}^{T} P_{b}\right)^{T}\left(L_{e q}^{T} P_{b}\right) \\
\mathbf{a}+\sum_{I P} w_{6}^{2}\left(\frac{\Delta t}{\gamma_{w}}\right)^{2}\left[k_{x} \frac{\partial^{2} P_{b}}{\partial \mathrm{x}^{2}}+k_{y} \frac{\partial^{2} P_{b}}{\partial \mathrm{y}^{2}}\right]^{T}\left[k_{x} \frac{\partial^{2} P_{b}}{\partial \mathrm{x}^{2}}+k_{y} \frac{\partial^{2} P_{b}}{\partial \mathrm{y}^{2}}\right]
\end{array}\right) \mathbf{b} \\
& =\left[\sum_{p \text { nnod }} w_{3}^{2} P_{b}^{T} \sigma_{p p}+\sum_{\text {bnod }} w_{4}^{2}\left(n P_{b}\right)^{T} \sigma_{b}+\sum_{I P} w_{5}^{2}\left(L_{e q}^{T} P_{b}\right)^{T} \omega\right]
\end{aligned}
$$

The above system can be written in matrix form as $[\mathbf{A}]\{\mathbf{X}\}=\{\mathbf{B}\}$ :

$$
\left[\begin{array}{ll}
A_{11} & A_{12} \\
A_{21} & A_{22}
\end{array}\right]\left\{\begin{array}{l}
\mathbf{a} \\
\mathbf{b}
\end{array}\right\}=\left\{\begin{array}{l}
B_{1} \\
B_{2}
\end{array}\right\} \text { where matrix orders are }\left[\begin{array}{cc}
2 n_{a} \mathrm{x} 2 n_{a} & 2 n_{a} \mathrm{x} n_{b} \\
n_{b} \mathrm{x} 2 n_{a} & n_{b} \mathrm{x} n_{b}
\end{array}\right]\left\{\begin{array}{c}
2 n_{\mathrm{a}} \\
n_{\mathrm{b}}
\end{array}\right\}=\left\{\begin{array}{c}
2 n_{\mathrm{a}} \\
n_{\mathrm{b}}
\end{array}\right\}
$$

$n_{\mathrm{a}}$ and $n_{\mathrm{b}}$ are the number of terms in the assumed polynomials $P_{\mathrm{a}}$ and $P_{\mathrm{b}}$ respectively. Being the method of choice for solving least-squares problems, the singular value decomposition (SVD) method is used to find $\mathbf{a}$ and $\mathbf{b}$ in preference to other equation-solving methods. These vectors are then substituted into the equations for $\mathrm{u}^{*}, \varepsilon^{*}$ or $\sigma^{* *}$ and $\sigma_{p p}^{*}$ respectively to obtain improved values. One point to note is that the terms $k_{x} \partial^{2} \sigma_{p p} / \partial \mathrm{x}^{2}$ and $k_{y} \partial^{2} \sigma_{p p} / \partial \mathrm{y}^{2}$ from equation (12) become incremental for nonlinear analyses, i.e. should be $\Delta\left(k_{x} \partial^{2} \sigma_{p p} / \partial \mathrm{x}^{2}\right)$ and $\Delta\left(k_{y} \partial^{2} \sigma_{p p} / \partial \mathrm{y}^{2}\right)$ respectively, where $\Delta f=f_{t+\Delta t}-f_{t}$. This is done by taking $\Delta \mathrm{f}$ between two increments or two groups of increments.

\subsection{Modified a-posteriori error estimators}

The importance of pore pressures in soil mechanics and the introduction of an improved function necessitate a change in the way that errors are calculated. In contrast to the energy norm, the $L_{2}$ norm is independent of the $\mathrm{D}$ matrix. This avoids the problem of 
successively smoothing the latter every increment for elasto-plastic applications, where the D matrix is a function of the stresses.

For the case of consolidation problems, the overall percentage displacement and pore pressure errors, $\eta_{\mathrm{u}}$ and $\eta_{\mathrm{pp}}$ in equations (15a) and (15b) respectively, are calculated based on the global displacement and pore pressure $L_{2}$ norms for all elements. The final overall percentage error $\eta$ is taken as the maximum of $\eta_{\mathrm{u}}$ and $\eta_{\mathrm{pp}}$. This is used to determine whether the results from the current mesh are within the specified tolerance $\eta \leq \bar{\eta}$, or whether further refinement is warranted.

$$
\begin{gathered}
\eta_{u}=\frac{\left\|e_{u}\right\|}{\left\|u_{u}\right\|}=\frac{\sum_{i=1}^{N E L} \sqrt{\int_{\Omega_{i}}\left(u^{*}-u\right)^{T}\left(u^{*}-u\right) d \Omega_{i}}}{\sum_{i=1}^{N E L} \sqrt{\int_{\Omega_{i}} u^{T} u d \Omega_{i}}} \\
\eta_{p p}=\frac{\left\|e_{p p}\right\|}{\left\|u_{p p}\right\|}=\frac{\sum_{i=1}^{N E L} \sqrt{\int_{\Omega_{i}}\left(\sigma_{p p}^{*}-\sigma_{p p}\right)^{T}\left(\sigma_{p p}^{*}-\sigma_{p p}\right) d \Omega_{i}}}{\sum_{i=1}^{N E L} \sqrt{\int_{\Omega_{i}} \sigma_{p p}^{T} \sigma_{p p} d \Omega_{i}}}
\end{gathered}
$$

In order to be able to create an optimal mesh, i.e. a mesh where the errors are equally distributed across the mesh, average displacement and pore pressure errors are defined as $e_{a v}^{u}=\bar{\eta} \sqrt{\left(\left\|u_{u}\right\|^{2}+\left\|e_{u}\right\|^{2}\right) / N E L}$ and $e_{a v}^{p p}=\bar{\eta} \sqrt{\left(\left\|u_{p p}\right\|^{2}+\left\|e_{p p}\right\|^{2}\right) / N E L}$ respectively, where $N E L$ is the number of elements in the mesh.

$$
\begin{gathered}
\xi_{i}^{u}=\frac{\left\|e_{u}\right\|_{i}}{\bar{\eta} \sqrt{\left(\left\|u_{u}\right\|^{2}+\left\|e_{u}\right\|^{2}\right) / N E L}}=\frac{\left\|e_{u}\right\|_{i}}{e_{a v}^{u}} \\
\xi_{\mathrm{i}}^{\mathrm{pp}}=\frac{\left\|e_{p p}\right\|_{i}}{\bar{\eta} \sqrt{\left(\left\|u_{p p}\right\|^{2}+\left\|e_{p p}\right\|^{2}\right) / N E L}}=\frac{\left\|e_{p p}\right\|_{i}}{e_{a v}^{p p}}
\end{gathered}
$$

For each element $i$, the ratio $\xi_{i}$ of the two variables, defined in equation (16), and an a-priori 
asymptotic convergence estimate are used to predict the new element size $h_{i}^{\text {new }}$ from the old size $h_{i}^{\text {old }}$. The values of $\xi_{i}$ provide an estimate of the amount of displacement and pore pressure errors in each element when compared with the overall average mesh errors, whereby values of $\xi_{i}$ greater than one indicate the need for element $i$ 's enlargement and viceversa. The new element size $h_{i}$ is chosen as the minimum of both values in equation (17), where $p$ is the order of the displacement elements used.

$$
\left(h_{i}^{\text {new }}\right)_{u}=\frac{h_{i}^{\text {old }}}{\sqrt[p+1]{\xi_{i}^{u}}} \text { and }\left(h_{i}^{\text {new }}\right)_{p p}=\frac{h_{i}^{\text {old }}}{\sqrt[p]{\xi_{i}^{\text {pp }}}}
$$

\subsection{Modifications for non-consolidation problems}

For non-consolidation problems, a different functional is defined, where time is not an issue and only the equilibrium equations govern the response. As a result, displacements are the only nodal degrees of freedom. The difference from a standard structural mechanics problem lies in the extra pore pressure parameter, which is equal to the in-situ pore pressures if the analysis is drained, and the product of the volumetric strain and water's bulk modulus $\mathrm{K}_{\mathrm{w}}$ for an undrained analysis. For the latter, Britto and Gunn [11] suggest that $\mathrm{K}_{\mathrm{w}}$ be taken as a value between 50 and 500 times the drained soil bulk modulus $\mathrm{K}^{\prime}$. This range corresponds to Poisson's ratio $v$ being between 0.49 and 0.499 respectively, where a value of $v$ equal to 0.5 leads to incompressible behaviour. Only a best-fit displacement polynomial is thus required, where $u^{*}=P_{a} \mathbf{a}$, and $P_{\mathrm{a}}$ is one order higher than the assumed displacement shape functions as before. Using section 2.2's notation, the functional $S$ becomes:

$$
\begin{aligned}
S= & \frac{1}{2}\left[\sum_{I P} w_{1}^{2}\left(\sigma^{\prime *}-\sigma^{\prime}\right)^{2}+\sum_{\text {unod }} w_{2}^{2}\left(u^{*}-u\right)^{2}+\sum_{I P} w_{3}^{2}\left(\sigma_{P P}^{*}-\sigma_{p p}\right)^{2}+\sum_{\text {bnod }} w_{4}^{2}\left(n \cdot \sigma^{*}-\sigma_{b}\right)^{2}\right. \\
& \left.+\sum_{I P} w_{5}^{2}\left(L_{e q}^{T} \sigma^{*}+\omega\right)^{2}\right]
\end{aligned}
$$

From the above functional, it can be observed that the pore pressure residuals are 
calculated at the integration points (or optimal points for triangles), and not at the pore pressure nodes as done previously. This is due to the pore pressures now being a function of the volumetric strain. The weightings used are the same as before, but one should note that $\mathrm{w}_{3}$ is equal to zero for drained analyses.

Assuming $u^{*}=P_{a} \mathbf{a}$, with $\sigma^{\prime^{*}}=$ Ma where $\mathrm{M}=\mathrm{DL}_{\varepsilon} P_{a}, \sigma^{*}={\sigma^{\prime *}}^{*} \sigma_{p p}^{*}$, and the volumetric strain $\varepsilon_{\mathrm{v}}$ equal to $K_{w} m^{T} \mathrm{~L}_{\varepsilon} P_{a} \mathrm{a}$ where $\mathrm{K}_{\mathrm{w}}$ is the bulk modulus of water previously discussed, $S$ becomes :

$$
\begin{aligned}
S= & \frac{1}{2}\left[\sum_{I P} w_{1}^{2}\left(M \mathbf{a}-\sigma^{\prime}\right)^{2}+\sum_{\text {unod }} w_{2}^{2}\left(P_{a} \mathbf{a}-u\right)^{2}+\sum_{I P} w_{3}^{2}\left(K_{w} m^{T} L_{\varepsilon} P_{a} \mathbf{a}-\sigma_{p p}\right)^{2}\right. \\
& \left.+\sum_{\text {bnod }} w_{4}^{2}\left(n\left(M \mathbf{a}+K_{w} m^{T} L_{\varepsilon} P_{a} \mathbf{a}\right)-\sigma_{b}\right)^{2}+\sum_{I P} w_{5}^{2}\left(L_{e q}^{T}\left(M \mathbf{a}+K_{w} m^{T} L_{\varepsilon} P_{a} \mathbf{a}\right)+\omega\right)^{2}\right]
\end{aligned}
$$

Minimising $S$ with respect to the unknown coefficient vector a and collecting terms:

$$
\begin{aligned}
& \left(\begin{array}{l}
\sum_{I P} w_{1}^{2} M^{T} M+\sum_{\text {unod }} w_{2}^{2} P_{a}^{T} P_{a}+\sum_{I P} w_{3}^{2}\left(K_{w} m^{T} L_{\varepsilon} P_{a}\right)^{T}\left(K_{w} m^{T} L_{\varepsilon} P_{a}\right)+\sum_{\text {bnod }} w_{4}^{2}\left(n\left(M+K_{w} m^{T} L_{\varepsilon} P_{a}\right)\right)^{T}\left(n\left(M+K_{w} m^{T} L_{\varepsilon} P_{a}\right)\right) \\
+\sum_{I P} w_{5}^{2}\left(L_{e q}^{T}\left(M+K_{w} m^{T} L_{\varepsilon} P_{a}\right)^{T}\left(L_{e q}^{T}\left(M+K_{w} m^{T} L_{\varepsilon} P_{a}\right)\right)\right.
\end{array}\right) \mathbf{a ~ ( 2 0 )} \\
& =\left[\sum_{I P} w_{1}^{2} M^{T} \sigma^{2}+\sum_{\text {unod }} w_{2}^{2} P_{a}^{T} u+\sum_{I P} w_{3}^{2}\left(K_{w} m^{T} L_{\varepsilon} P_{a}\right)^{T} \sigma_{p p}+\sum_{\text {bnod }} w_{4}^{2}\left(n\left(M+K_{w} m^{T} L_{\varepsilon} P_{a}\right)\right)^{T} \sigma_{b}+\sum_{I P} w_{5}^{2}\left(L_{e q}^{T}\left(M+K_{w} m^{T} L_{\varepsilon} P_{a}\right)^{T} \omega\right]\right.
\end{aligned}
$$

The above system can be written in matrix form as

$$
[A]\{\mathbf{a}\}=\{B\} \text { where matrix orders are: }\left[2 n_{a} \times 2 n_{a}\right]\left\{2 n_{\mathrm{a}}\right\}=\left\{2 n_{\mathrm{a}}\right\}
$$

As before, $n_{\mathrm{a}}$ is the number of terms in the assumed polynomial $\mathrm{P}_{\mathrm{a}}$. This set of matrices is solved for the vector a using the SVD decomposition method. The vector $\mathbf{a}$ is then substituted into the equations for $\mathrm{u}^{*}, \varepsilon^{*}$ or $\sigma^{\prime *}$, and if the analysis is undrained, $\sigma_{p p}^{*}$. Only the $\mathrm{L}_{2}$ displacement errors need to be computed and used as a criteria for further refinement. This is obvious for a drained analysis. For undrained analyses, the displacements, of which pore pressures are a function, are the primary variable being improved, and so it is logical to try to quantify the errors based on this primary variable alone. 


\subsection{Procedure and implementation}

The CSPREB method works in a similar way to the patch recovery methods, albeit a few differences due to the extra parameters involved. For each vertex node in the mesh, the elements which share the node are found. If the number of elements in the patch is less than three, then this vertex node is discarded and the next vertex node is selected due to the lack of sufficient data points for a reliable best-fit. One should note that unlike SPR-based methods, the variables being smoothed in the CSPREB method are displacements and pore pressures, which are equal across boundaries of different materials. Hence, no special measures have to be taken when elements of different materials occur in the same patch. However after the displacements and pore pressures have been determined, variables at integration points and across boundaries are calculated for each element separately.

For each element in the patch, the various quantities in the matrix $[\mathbf{A}]$ and vector $\{\mathbf{B}\}$ from equations (14) or (21) are calculated by looping over the various nodes and optimum points. The vector $\{\mathbf{X}\}$ is then found using the SVD method, and the displacements $u^{*}=P_{a} \mathbf{a}$ and pore pressures $\sigma_{p p}^{*}=P_{b} \mathbf{b}$ are calculated at the integration points. An important issue arises here with respect to the simultaneous equations. The least squares method is notorious for producing ill-conditioned systems of equations especially in regions of high variations of sampling data points. One way of circumventing this problem is to map the local patch parametric space onto a rectangle that encloses the patch. This means that the rectangular coordinates $(\mathrm{x}, \mathrm{y})$ would map to the local co-ordinates $(\xi, \eta)$, where the latter vary from -1 to 1 . Zienkiewicz et al. [15] used a similar approach to normalise the co-ordinates and Wiberg et al. [7] used different co-ordinate transformations for the same purpose. The transformations used here are given by equation (22), where $\left(\mathrm{x}_{\max }, \mathrm{y}_{\max }\right)$ and $\left(\mathrm{x}_{\min }, \mathrm{y}_{\min }\right)$ are the maximum and minimum co-ordinates in the patch respectively. 


$$
\xi=-1+\frac{2\left(x-x_{\min }\right)}{\mathrm{a}}, \eta=-1+\frac{2\left(y-y_{\min }\right)}{\mathrm{b}}, \begin{aligned}
& \mathrm{a}=x_{\max }-x_{\min } \\
& \mathrm{b}=y_{\max }-y_{\min }
\end{aligned}
$$

Figure 2 depicts a typical patch inside the local co-ordinate system limits. The transformation of co-ordinates introduces the necessity of multiplying derivatives by Jacobians when integrating the various quantities during the assembly of the simultaneous equations. By using the chain rule, the Jacobians, by which the various derivatives are multiplied, are given in equation (23).

$$
\begin{array}{lll}
u_{, x}=(2 / a) u_{, \xi} & u_{, x x}=\left(4 / a^{2}\right) u_{, \xi \xi} \\
u_{, y}=(2 / b) u_{, \eta} & u_{, x y}=(4 / a b) u_{, \eta \xi} & u_{, y y}=\left(4 / b^{2}\right) u_{, \eta \eta}
\end{array}
$$

After displacements and pore pressures have been calculated for all the patches, the elements are processed to compute the $\mathrm{L}_{2}$ displacement and pore pressure norms. Inevitably, there will be points where several patches are used to calculate their respective values; in these cases, an average of the patch values is used. Assuming the norms do not satisfy the specified global percentage tolerance, the new element sizes are calculated based on the norms to generate a completely new mesh. Otherwise, the results obtained from this last analysis are those which are taken as final values.

For non-linear materials or consolidation-based problems, the solution has to be computed incrementally. The a-posteriori errors may be evaluated at the end of each increment or fixed number of increments, or at the end of the final increment of the analysis. An example of the former may be found in Mar [2], where remeshing occurred every 5 increments. However, the mapping of variables from the old mesh to the new one would consume a large amount of time, and the associated complexities would make adaptive mesh refinement inefficient. Fixing the number of increments at which refinement occurs would also be disadvantageous since refining the mesh may be required at a stage during the fixed number of increments rather than at the start or end. The authors have opted to refine at the 
end of "increment blocks", whereby a variable increment block comprises any number of increments, depending on the problem in hand.

\section{Numerical examples}

In this section, two examples are presented to demonstrate the effectiveness of the criterion. For the first example, the deviation of the solutions of the resulting meshes from closed-form solutions are examined. A very fine mesh is used in lieu of a closed-form solution for the second example.

\subsection{Mandel Problem}

In this section, the consolidation aspect of the criterion, through the combination of displacement and pore pressure improvement algorithms, is validated by means of the Mandel problem [16]. A load $p$ of $100 \mathrm{kPa}$ is applied to a plane strain elastic weightless clay layer, having impermeable upper and lower horizontal boundaries and permeable vertical boundaries. Half of the domain is modelled due to symmetry, resulting in the initial uniform mesh shown in figure 3, having square plane dimensions $a$ and 315 degrees of freedom (dof). The horizontal and vertical permeabilities are both $10^{-8} \mathrm{~m} / \mathrm{s}$, Poisson's ratio $v$ is 0.1 and the soil's modulus of elasticity $\mathrm{E}$ is $10^{4} \mathrm{kPa}$. The Mandel problem which has a closed-form solution for comparison, is used to validate the CSPREB criterion, through the combination of the displacement and pore pressure improvement algorithms.

Equation (24) provides a closed-form solution during time $t$ along the horizontal distance from the centreline $x$, derived by Mandel for the excess pore pressures generated in the ground. In this equation, $\mu$ and $\lambda$ are Lamé's coefficients, while $c_{v}$ is the consolidation coefficient, defined by equation (24c), where $k$ and $\gamma_{\mathrm{w}}$ are the soil permeability and unit weight of water respectively.

$$
\sigma_{p p}=\sum_{i=1}^{\infty} A_{i}\left(\cos \left(\frac{\alpha_{i} X}{a}\right)-\cos \alpha_{i}\right) e^{-\left(\frac{\alpha_{i}^{2} c_{v} t}{a^{2}}\right)}
$$




$$
\begin{aligned}
\text { where } A_{i} & =\frac{p(\lambda+2 \mu) \cos \alpha_{i}}{\mu-(\lambda+2 \mu) \cos ^{2} \alpha_{i}} \text { and } \alpha_{\mathrm{i}} \text { are roots of } \tan \alpha=\left(\frac{\lambda+2 \mu}{\mu}\right) \alpha \\
\lambda & =\frac{E v}{(1+v)(1-2 v)}, \mu=\frac{E}{2(1+v)} \text { and } c_{v}=\frac{k(\lambda+2 \mu)}{\gamma_{w}}
\end{aligned}
$$

The soil in figure 3 is loaded at the start of the analysis by a load $p$, causing pore pressure gradients to form. The soil consolidates causing water to flow laterally towards the permeable boundary on the right hand-side of the mesh. The analysis was split into 90 equal sized increments, covering $90 \%$ of the duration of the consolidation process, which is of most interest to geotechnical engineers. The first stage of refinement, for a specified tolerance $\eta_{\text {tol }}$, occurred when a very large pore pressure gradient formed at the permeable boundary, which was at approximately $\mathrm{T}_{\mathrm{v}}=4.17 \times 10^{-4}$. After remeshing, the accuracy of the new mesh was checked and the analysis resumed. Refinement was then performed at the end of the analysis using a tolerance of $0.5 \%$, when all pore pressures had dissipated.

Figure 4(a) shows the mesh generated after the first stage. As anticipated, the permeable boundary is heavily refined due to high pore pressure gradients there. At this early stage of the analysis, where the soil has had little time to deform significantly, a negligible improvement in horizontal and vertical displacement contours due to refinement was observed. Figures 5(a) and 5(b) depict the pointwise displacements at this stage, where $(0,0,0)$ in the $3 \mathrm{D}$ plots represents the bottom left-hand corner of the mesh. Although the shape of these graphs might raise some concern, the actual scale of error is very small when compared with the magnitude of displacements, i.e. about one thousandth of the actual displacements. However, the pore pressure contours in figures 6(a) and 6(b), and pointwise errors in figures 7(a) and 7(b) show a different story. The contour profiles have undergone a major change in character, largely due to the high errors at the permeable boundary incurred by the high pore pressure gradients. The latter zone has become much narrower, with the 
distinctive jump in the pore pressure errors' surface at the right hand-side in figures 7(a) and 7(b) flattening out. Even though $\mathrm{e}_{\mathrm{pp}}$ in figure 7(b) has not been reduced to a value close to zero, the main objective of equalising all errors across a mesh has been achieved within the percentage tolerance specified.

During the first stage of analysis, the pore pressures had dictated where refinement would occur. However, by the end of the analysis, all pore pressures had dissipated, playing a minor role in deciding where refinement is to occur, while displacements became the main factor controlling refinement. Notwithstanding, the pore pressure errors at the final stage were less than the $\eta_{\text {tol }}=5 \%$ of the first stage, so a tolerance of $0.5 \%$ that would guarantee refinement based on displacements and pore pressures was specified. Even though the number of degrees of freedom in figure 4(b) is not significantly higher than the mesh in figure 4(a), the distribution of element sizes has changed considerably, with larger elements existing in place of what was previously a region of high pore pressure. A slightly finer portion of the mesh exists at the left edge where the pore pressures and errors are higher than the rest of the mesh, as shown in figure 7(c). In figure 5(c), the upper loaded edge of the mesh is more refined due to the existence of the highest vertical displacements and errors there. A smaller patch in the upper right-hand corner of the mesh is also fine, but to a lesser degree, due to a combination of maximum horizontal and vertical displacements. The pore pressure errors' surface in figure 7(c) has also decreased in slope to become flatter, as shown in figure 7(d).

Figures 8(a) and 8(b) show the normalised pore pressure variations across a horizontal section as the consolidation progresses for the initial and final meshes respectively. At time $t=0$, upon applying the load, the pore pressures jump everywhere to a value equal to half the applied load. The pore pressures then start to increase at the left hand-side region of the mesh, while decreasing in other areas. As time passes and lateral consolidation occurs, the 
pore pressures dissipate. This feature involving an initial jump followed by a decrease in pore pressures is known as the Mandel effect. Figure 9 shows the history of pore pressures with time and demonstrates this effect clearly. Remeshing has brought the normalised pore pressures into virtual agreement with the theoretical curve in figure 9, while the Mandel effect is much more pronounced. The maximum normalised pore pressure $\sigma_{\mathrm{pp}} / \mathrm{p}$ increased from a value of 0.533 for the initial mesh to 0.563 for the final mesh due to remeshing, which is very near the closed-form solution of 0.567 . The time at which these maximum pore pressures occurred has also moved closer from the square root of $T_{v}$ equal to 0.189 for the initial mesh to the square root of $T_{v}$ equal to 0.266 for the regenerated mesh, which is the same as the exact solution.

Timewise, the whole analysis, remeshing, and post-processing consumed 56.6 minutes on a Pentium-90 processor. In contrast, assuming that the analyst has no prior knowledge of the areas requiring refinement, a very fine mesh constituting 10106 dof required 141 minutes, even though the latter mesh was used to satisfy a higher tolerance of $5 \%$.

\subsection{Smooth rigid strip footing on an undrained double-layered Tresca material}

To demonstrate the use of the non-consolidation formulation, a rigid smooth strip footing applying a surcharge $q$ on a two-layered soil, is analysed under undrained conditions. The collapse load is then compared with plasticity bounds, and results from a very fine mesh. Various solution methods exist for single-layered soil problems. However, the majority of real-life problems involve multi-layered soils, where properties vary widely and deriving an analytical collapse load solution becomes very complicated. In addition to empirical methods, numerical prediction can therefore occupy a supporting role in estimating failure loads.

Half the problem is modelled due to symmetry, resulting in the initial mesh depicted in figure 10, having 502 degrees of freedom. The soil is assumed to be weightless and yields 
according to the Tresca criterion. The lower soil layer has a constant shear strength $\mathrm{S}_{\mathrm{u} 2}$ twice the shear strength of the upper layer $\mathrm{S}_{\mathrm{u} 1}$ of $10 \mathrm{kPa}$. Figure 11(a) shows the refined mesh at the onset of yielding, with an enlarged view of the footing region. The deviator stress contours for this stage in figures 12(a) and 12(b) have experienced very significant changes in shape, especially underneath the footing, where the stresses have increased in magnitude. The large jump in pointwise displacement errors in figures 13(a) and 13(b) at the edge of the footing has also disappeared after refinement.

Figure 11(b) depicts the final mesh after complete yielding. The enlarged view of the very fine elements in figure 11(d) shows how the top layer has completely yielded, while only small areas in the lower layer have been affected. The lower layer has thus acted as a solid bedrock, forcing the upper soil to flow laterally in a similar fashion to the toothpaste lateral extrusion problem. The deviatoric stress contours for this stage, have also improved, but this change is not as drastic as for the initial stage, due to the region below the footing being already refined.

Figure 14 shows the bearing capacity curves, where the bearing capacity factor $\mathrm{N}_{\mathrm{c}}=\mathrm{q} / \mathrm{S}_{\mathrm{u} 1}$. Refining the initial mesh resulted in the bearing capacity factor decreasing from an initial value of 6.59 to 5.63. A very fine mesh comprising 10214 degrees of freedom yielded a bearing capacity factor of 5.64, which took approximately three and a half times longer than using remeshing. These values are also in accord with the range of $4<\mathrm{N}_{\mathrm{c}}<5.76$ [14] for such a problem.

\section{Conclusions}

The crux of good soil modelling is the constitutive law used, which usually depends on the stress history and stress path of the geomechanical process. For soil models which behave plastically, erroneous effective stress states result in wrongly predicting the soil behaviour at and after yield, such as when the soil is either hardening or softening. It is 
essential to control discretization errors in nonlinear systems. Adaptive mesh refinement with the new error criterion has achieved this via the use of the various weights and residuals to regenerate different meshes at different stages of geotechnical analyses.

Adaptive mesh refinement was found to be very efficient in improving the prediction of soil behaviour for plane strain problems. The consolidation problem clearly demonstrated the importance of including pore pressures in the error estimation process, which allowed element sizes of regions with varying activity first to decrease then increase according to how the gradients changed. From solving various problems [14], the time consumed has been reduced to about half to a third of the time taken to analyse a uniformly fine mesh for the same accuracy level. The use of AMR therefore has significant advantages in general finite element analyses, such as for multi-layered soils, where exact solutions are not known. The time advantage would become much more critical when dealing with three-dimensional problems, where use of a fine uniform mesh would be impractical and inefficient. Finally, for the two examples shown, and others used to validate the criterion [14], an ultraconvergent rate was observed which was approximately two orders higher than the global rate. The improved accuracy by incorporating pore pressures into the error estimation process, and derived benefits, would thus enable analysts to acquire a better understanding of the soilstructure interaction.

\section{References}

[1] X. R. Wang, D. H. Chan, and N. R. Morgenstern, A numerical scheme for modelling kinematic propagation of shear bands, in: Proceedings of the 5th International Symposium of Numerical Methods in Geomechanics, Davos (Switzerland), pp. 215222,1995.

[2] A. Mar, Adaptive mesh refinement for nonlinear problems in geomechanics using an advanced constitutive law, Ph.D. Thesis, University of Manchester, Manchester 
(U.K.), 1993.

[3] O. C. Zienkiewicz and J. Z. Zhu, “A simple error estimator and adaptive procedure for practical engineering analysis”, International Journal for Numerical Methods in Engineering, 24, pp. 337-357, 1987.

[4] O. C. Zienkiewicz, M. Huang, and M. Pastor, "Localisation problems in plasticity using finite elements with adaptive remeshing”, International Journal for Numerical and Analytical Methods in Geomechanics, 19, pp. 127-148, 1995.

[5] M. A. Hicks, Computation of localisation in undrained soil using adaptive mesh refinement, in: Proceedings of the 5th International Symposium of Numerical Methods in Geomechanics, Davos (Switzerland), pp. 203-208,1995.

[6] O. C. Zienkiewicz and J. Z. Zhu, "The superconvergent patch recovery and $a$ posteriori error estimates (Parts 1 and 2)”, International Journal for Numerical Methods in Engineering, 33, pp. 1331-1382, 1992.

[7] N. E. Wiberg, F. AbdulWahab, and S. Ziukas, "Enhanced superconvergent patch recovery incorporating equilibrium and boundary conditions”, International Journal for Numerical Methods in Engineering, 37, pp. 3417-3440, 1994.

[8] B. Boroomand and O. C. Zienkiewicz, "Recovery by equilibrium in patches (REP)", International Journal for Numerical Methods in Engineering, 40, pp. 137-164, 1997.

[9] M. A. Biot, “General theory of three-dimensional consolidation”, Journal of Applied Physics, 12, pp. 155-164, 1941.

[10] R. S. Sandhu and E. L. Wilson, "Finite element analysis of seepage in elastic media”, Journal of Engineering Mechanical Division, 95, pp. 641-652, 1969.

[11] A. M. Britto and M. J. Gunn, Critical state soil mechanics via finite elements, Ellis Horwood Ltd., Chichester (UK), 1987.

[12] J. R. Booker and J. C. Small, “An investigation of the stability of numerical solutions 
of Biot's equations of consolidation”, International Journal of Solids and Structures, 11, pp. 907-911, 1975.

[13] N. E. Wiberg and F. AbdulWahab, "Patch recovery based on superconvergent derivatives and equilibrium”, International Journal for Numerical Methods in Engineering, 36, pp. 2703-2724, 1993.

[14] A. El-Hamalawi, Adaptive refinement of finite element meshes for geotechnical analyses, Ph.D. Thesis, Engineering Department, Cambridge University, Cambridge (U.K.), 1997.

[15] O. C. Zienkiewicz, J. Z. Zhu, and J. Wu, "Superconvergent patch recovery techniques - some further tests”, Communications in Numerical Methods in Engineering, 9, pp. 251-258, 1993.

[16] J. Mandel, “Consolidation des sols (étude mathématique)”, Geotechnique, 3, pp. 87299, 1953. 